\title{
Public and Private Investments in Regulated Network Industries: Coordination and Competition Issues*
}

\author{
Bruno Jullien ${ }^{\dagger}$ Jerome Pouyet ${ }^{\ddagger}$ Wilfried Sand-Zantman ${ }^{\S}$ \\ July 28, 2009 \\ Very preliminary. Comments welcome.
}

\begin{abstract}
This exploratory paper analyzes the three-way relationship between a national regulator, an incumbent and a local authority in a context where investment in a new infrastructure has to be undertaken. This setting is relevant for the analysis of local investment in next generation access network in telecoms.

It is optimal to allow duplication by the local authority when the incumbent can perfectly anticipate the local authority's investment decision, national and local preferences coincide and there is no externality between localities.

When the incumbent lacks information about the local authority's decision, it faces a risk of duplication. The regulator can either allow duplication, but must then compensate the incumbent through a higher access tariff, or ban duplication. The latter is shown to dominate the former.

The advantage of a ban on duplication are less clear when local and national objectives are not aligned and we discuss the merits of various other remedies such as authorizations or compensation to the incumbent.

We finally discuss the impact of externalities between localities, such as migrations or returns to scale.
\end{abstract}

\section{Introduction}

While network industries have been open to competition by now, public intervention remains pregnant, be it in the form of access regulation or universal service obligations to name but

*We gratefully acknowledge France Télécom for its intellectual and financial support.

${ }^{\dagger}$ Toulouse School of Economics.

${ }^{\ddagger}$ Paris School of Economics and CEPR.

$\S$ Toulouse School of Economics. 
a few. Moreover, private actors in these industries must undertake substantial long-term infrastructure investments in order to gain a competitive edge over their rivals by offering higher-quality services to customers. The profitability of these investments may be affected by other variables, though. First, as hinted previously, new infrastructure networks, which typically involve a long-term planing of the coverage deployment, may be regulated by a national authority. Second, public investment may cannibalize the profitability of private investment; local or regional authorities may indeed consider preemptive investment schemes to ensure the accelerated delivery of new services. This paper analyzes such a three-way interaction between a national regulator, private actors and a local authority, in a context where both the private sector and the local authority may invest.

As an example of primary interest, consider the telecommunications industry. Next generation access networks will allow to reach higher delivering speeds than ADSL2+ or cable technologies. Despite the uncertainty surrounding the demand for these new services, many decision-makers foresee in these investments the potential for a profound impact on the broadband market, but also on society. ${ }^{1}$ Investment costs for the deployment of these networks and the upgrading of the existing network are substantial, though, and entail some risks for private actors. In this context, some local public authorities or regional development agencies have decided to build their own infrastructure in order to boost the delivery of new services to their constituencies.

There are certainly valid reasons to allow, or sometimes encourage, these local authorities to develop their own network, among which market failures, distributional objectives including equity and social inclusion, or regional competitiveness. However, as recognized by Ofcom (2007b), ARCEP (2008) or EC (2009), there are several problems which need careful attention. Our paper explores some of these problems.

We build a canonical model of the relationship between a national regulator, a local authority and a private actor. The national territory is divided into 'districts', which differ by the level of demand for new services and the cost of building a new network.

An incumbent operator contemplates the decision whether to invest in a new infrastructure in each district. Such an infrastructure can be rented to a competitive fringe of operators to provide services to customers. For the incumbent to be willing to invest, some access markup is needed to cover the investment cost. The regulator is in charge of regulating the access to the incumbent's network.

The local authority can decide to develop its own network infrastructure, which then decides the terms of access to the local network. The local authority is interested in the welfare of its constituency and, in particular, can use local public funds to finance the network, as opposed to the regulator. We assume that the local authority is a priori less efficient than the incumbent in building the new network, but since the former can use public funds it may also set a lower access charge on its network, thereby improving customers' surplus.

The sequence of decisions is as follows. First, the regulator sets the access charge which

\footnotetext{
${ }^{1}$ See Ofcom (2007a) for instance.
} 
applies to the incumbent's network on the national territory. Second, the incumbent decides whether to invest. Third, the local authority may invest in, and decide the terms to access of, a local network. Our goal is to determine the optimal regulatory regime, that is, whether the local authority should be allowed to or banned from investing in a public network. ${ }^{2}$

Assume in a first step that the local authority's cost of investment is publicly known. Then, we show that one should always allow the local authority to invest. The reason is straightforward: under complete information, the incumbent can perfectly anticipate the local authority's decision and there is no inefficient duplication. The regulator, in turn, can set a regulated access tariff which leaves the incumbent with no extra profit, implying that the local authority's objective becomes aligned with the regulator's. In a nutshell, delegation of the investment decision has no social costs in this setting.

This result extends to more general situations provided that, on the one hand, the incumbent and the local authority share the same information, and, on the other hand, the regulator and the local authority share the same objective. If one of these conditions is not satisfied, then the regulator may want to constraint the local authority's intervention.

Consider now the situation in which the regulator does not know the local authority's cost of investment. However, that information is known by the incumbent. Suppose, moreover, that the regulated access price, which is determined nation-wide, leaves some profits to the incumbent if it decides to invest in the district; this is the case, for instance, under the socalled 'perequation schemes' which require the access prices to be uniform across the whole territory.

The local authority's cost may not be known ex ante, but can be verifiable ex post. In that case, several regimes of authorization are possible. On top of systematically allowing duplication, the regulator can also decide on a case-by-case basis, i.e. ex post once the local authority's cost is known, whether the local authority is allowed to duplicate. A systematic authorization of duplication leads to excessive investment by the local authority, which does not internalize the incumbent's foregone profit; this deters some efficient investment by the incumbent. By contrast, a regime with ex post authorization yields too much private investment.

A different approach for the regulator consists in constraining the local authority's intervention. This may be best suited when the local authority's cost cannot be verified, both ex ante and ex post. The regulator may then decide to implement a regulation of the access to the local network as well as a reimbursement policy in the event of duplication. We isolate conditions under which these two constraints on the local authority's intervention may be optimal or not.

We also show that these policies are not unrelated. In particular, when a regime with ex post authorization prevails, then the incumbent should be compensated by the local authority when it duplicates.

\footnotetext{
${ }^{2}$ In our companion paper (Jullien, Pouyet and Sand-Zantman 2009) we investigate the possibility that the local authority reaches a contractual agreement with the incumbent, including subsidies, which in not allowed here.
} 
We then turn our attention to a situation in which neither the national regulator nor the incumbent are informed about the local authority's cost of intervention. Hence, the incumbent cannot perfectly foresee when it will be duplicated and is exposed to a risk. In this context, duplication is no longer always efficient and the regulator faces two options. A ban on duplication removes the duplication risk faced by the incumbent, but does not allow to benefit from reduced access tariff when the local authority invests. By contrast, allowing duplication forces the regulator to increase the regulated access price so as to compensate the incumbent from the duplication risk. A priori, both policies come with their own costs and benefits. However, we prove that, if there is private investment, then a ban on duplication is socially optimal.

We conclude our analysis with a brief consideration of externalities across districts. Two types of externalities can be envisioned. First, scale economies may make it less costly for the incumbent to deploy investment over the whole territory. Second, in presence of a mobile population, which patronizes one district over the other depending on the possibility to benefit from new services, districts create externalities on each others. This situation has some analogies with the literature on fiscal competition between local authorities. In these settings, allowing duplication has, again, costs and benefits.

Our paper belongs to the literature on regulation in network industries (see Laffont and Tirole, 2000, for instance). Our main departure is to focus on the interaction between a regulator and a local authority, each having specific attributes; hence, our paper also is related to the literature on the provision of local public goods. A few articles have discussed the regulation process when different market structures are possible. In the literature focusing on the regulation of infrastructures, the standard trade-off is between granting a generous access to the essential facility (or promoting competition at the upstream level) and recouping the cost of investment. For example, Dana and Spier (1994) made one of the first contributions where the modes of production and the market structure are endogenous. Even closer to our paper is the article of Caillaud and Tirole (2004) highlighting a conflict between social optimality and financial viability. We also have a similar conflict, but the potential competitor is a public agency. Moreover, we analyze the role of the national regulator in mitigating this risk by choosing the regulation rules.

The paper is organized as follows. The key ingredients of our model are exposed in Section 2. Section 3 analyzes the benchmark case of complete information. Section 4 studies the distortion generated by the lack of information on the regulator's side, whereas Section 5 focuses on the duplication risk that arises when the incumbent lacks some information about, and cannot predict, the local authority's intervention. Externalities are examined in Section 6. Section 7 concludes. 


\section{Model}

There is one representative geographical zone, called the 'district', characterized by its level of demand for broadband services denoted by $\theta$.

Customers located on the district may benefit from a new service provided by a set of identical firms. The provision of this service requires access to an up-to-date network. An incumbent operator, denoted by $I$, has the possibility to upgrade its existing network at a cost $c>0$ to allows the provision of the service. ${ }^{3}$

Access to the infrastructure network is set on a nondiscriminatory basis and the unit price is denoted by $a$. Service providers are assumed to behave competitively with a constant marginal cost normalized to zero, so that the final price they charge to customers is always equal to the access charge $p=a$. The demand is then $\theta D(a)$ and we assume $D(0)>0$. The corresponding consumers surplus is denoted by $\theta W(a)$, with $W^{\prime}(a)=-D(a)$. Let $\varepsilon(a)=$ $-a D^{\prime}(a) / D(a)$ be the price elasticity of the demand. We assume that $\varepsilon(a)$ is increasing and $\varepsilon(0)=0$. As this will always be optimal to set price below the monopoly level, we restrict attention to access price $a$ such that $\varepsilon(a) \leq 1$.

Access to the incumbent network is regulated. The regulator $R$ is in charge of the pricing of the access to the existing network and commits to an access charge $a=r$ prior to the decision of the incumbent to upgrade or not.

Instead of relying on the incumbent, a local authority $L$, representing the constituency of the district, may decide to build its own network. L's cost is given by $k$ and is distributed on $[\underline{k},+\infty)$, with $\underline{k} \geq c$, according to a strictly positive density $f($.$) and \operatorname{cdf} F($.$) . The local$ authority's objective is to maximize the welfare of its constituency.

The network built by $L$ is not subject to the access regulation that applies to $I$ 's network. However, in order to focus on the most relevant cases, we assume (without loss of generality) that $L$ cannot implement an access price higher than the regulated access price. If both the incumbent and the local authority builds an upgraded network, there is Bertrand competition on the wholesale market for access.

Finally, events unfolds as follows. First, the regulator $R$ decides of the price for the access $r$ to the existing network. Second, the value of $k$ is realized. Third, incumbent operator $I$ decides whether to upgrade or not its network. Fourth, local authority $L$ decides whether to build a competing network. If it does, then it can decide the term of access to the local public network newly created; if it doesn't, then broadband services might be provided by the firms using the already existing network at the access price $r$ decided by the regulator $R$.

The local authority is thus allowed to intervene and to duplicate the investment of $I$ or to invest when $I$ fails to do so. We refer to this regime as the regime $D$ (for duplication or duopoly). We will compare that scenario with two other possible regulatory regimes.

In regime $L$, the incumbent is not investing (because either regulation forbids investment or $r$ is low enough to discourage it) and $L$ decides to provide the service locally or not. The

\footnotetext{
${ }^{3}$ This cost may depend on the observable characteristics of the district such as its density for example.
} 
regime $M$ (for monopoly) is one in which the local authority is not allowed to duplicate the infrastructure. In this case, the local authority is allowed to invest in a network only if the incumbent has not. ${ }^{4}$

\section{Benchmark}

As a preliminary step, assume that the incumbent's and the local authority's investment cost are publicly known.

Let us first analyze the investment decisions by the incumbent and the local authority. At the last stage of the game, $L$ decides whether to duplicate the network. Obviously, that decision depends on the choice made by $I$ at the previous stage. If $I$ has not upgraded the network, then $L$ decides to build its own network if, and only if:

$$
\max _{a \leq r} \theta W(a)+\theta a D(a)-k \geq 0
$$

The left-hand side of (1) is the welfare of the local authority when it upgrades the network, and sets optimally the access price for that network. ${ }^{5}$ The optimal access price is thus equal to nil. Hence, condition (1) can then be rewritten as $\theta \geq \frac{k}{W(0)}$.

If $I$ has upgraded the network, then the local authority is willing to duplicate the network if, and only if:

$$
\max _{a \leq r} \theta W(a)+\theta a D(a)-k \geq \theta W(r) .
$$

The difference with inequality (1) is the gain for the local authority if it decides not to intervene and contents itself with broadband services being provided to its local constituency at final price $r$. Simple manipulations allow to rewrite inequality (2) as

$$
k \leq \hat{k}(r)=\theta[W(0)-W(r)]
$$

Let us now turn on to the decision faced by the local incumbent at the second stage of the game. The incumbent is not willing to undertake the upgrading if it expects to make losses from such a decision. Negative profits arise either because the level of demand for broadband services is too low to cover the fixed cost of the upgrading, or because the local authority decides later on to bypass the incumbent's network. Therefore, $I$ invests provided that:

$$
\frac{c}{r D(r)} \leq \theta \leq \frac{k}{W(0)-W(r)}
$$

Situations in which the incumbent never invests (either because it is not economically

\footnotetext{
${ }^{4}$ An alternative would be to forbid completely the intervention of $L$ but this would be dominated by regime $M$ since investment by $I$ would be the same, but $L$ would invest less.

${ }^{5}$ That welfare is the sum of customers' surplus and the access revenues generated by the local public network, minus the fixed cost of duplication $k$; it does not account for the incumbent's profit.
} 
viable or because the incumbent expects to be duplicated by the local authority) are of limited interested. Therefore, we focus on cases in which inequality (4) is satisfied. As established in the next lemma, we can safely consider that (4) holds for some values of the access price $r$ set by the regulator.

Lemma 1 There exists values of $(r, \theta)$ such that $\frac{c}{r D(r)}<\theta<\frac{k}{W(0)-W(r)}$ if and only if $k>c$.

Proof. This amounts to proving that, for some values of $r, \frac{c}{r D(r)}<\frac{k}{W(0)-W(r)}$ or equivalently that $h(r) \equiv W(r)+\frac{k}{c} r D(r)-W(0)>0$. We have: $h^{\prime}(r)=\frac{D(r)}{c}[k-c-k \varepsilon(r)]$. Since $\varepsilon($.$) is$ increasing, $h($.$) is quasi-concave in r$. Thus $h(r)$ is positive for some values of $r$ if and only if $h^{\prime}(0)=(k-c) D(0)>0$, or $k>c$.

Social efficiency solves the following trade-off. The incumbent is more efficient than the local authority to build the infrastructure. However, as opposed to the local authority, the regulator cannot use taxation to finance the investment and must distort the access price away from the marginal cost of access.

In a regime in which the incumbent invests, the access price $r_{b b}$ implemented by the regulator is such that the incumbent exactly breaks even, or: $\theta r_{b b} D\left(r_{b b}\right)=c$. Welfare is thus equal to: $\theta W\left(r_{b b}\right)$.

If, by contrast, the regulator lets the local authority undertake the investment, $L$ implements an access price which maximizes the welfare of its constituency, which as we have seen, leads to a nil access price. Welfare is thus equal to: $\theta W(0)-k$.

Therefore, the first-best investment rule is as follows: the incumbent invests at a regulated access price $r_{b b}$ if and only if $k \geq \hat{k}\left(r_{b b}\right)$; otherwise, the local authority invests and sets a nil access price on the new infrastructure.

In this framework where all the relevant information is available to all the actors, there are various ways to implement the first-best allocation. For instance, the regulator can dictate which party has to undertake the investment. Or, $R$ can let the local authority decide who has to make the investment (but $R$ keeps the power to set the access price if the incumbent builds the infrastructure).

Alternatively, suppose that $R$ provides the incumbent with the incentives to invest by setting an access price equal to $r_{b b}$, and allows the local authority to duplicate the incumbent's network. It is straightforward that this regulation triggers the socially optimal investment choice at equilibrium of our game, i.e. Regime $D$ implements the first-best. By contrast, both Regime $M$ (which bans duplication) and Regime $L$ (which prevents investment by the incumbent) are sub-optimal under complete information.

The next proposition summarizes this benchmark.

Proposition 1 First, under complete information, at the social optimum, if $k \geq \hat{k}\left(r_{b b}\right)$ then the incumbent invests and the regulated access price is $r_{b b}$; otherwise, the local authority invests and sets an access price equal to nil.

Second, allowing duplication (i.e. Regime D) implements the first-best allocation while Regime $L$ and $M$ are sub-optimal. 
Key to the second part of Proposition 1 is the fact that, under complete information, $L$ 's intervention is perfectly anticipated by $I$ and $R$ and that $R$ sets an access charge which leaves no profit to the incumbent so that $L$ 's and $R$ 's objectives become perfectly aligned. The next proposition shows that this logic extends to a more general informational setting.

Proposition 2 If, on the one hand, the regulator maximizes consumers' welfare, and, on the other hand, the incumbent and the local authority share the same information, then allowing duplication is socially preferable to any other regimes.

Proof. Consider any information structure such that $I$ and $L$ have the same information, denoted by $s$. The access charge chosen under regime $M$ is $r_{M}$. Suppose that the regulator chooses regime $D$ with $r=r_{M}$. Then, $I$ invests when $\mathbb{E}\{\theta W(0)-k \mid s\} \leq \mathbb{E}\left\{\theta W\left(r_{M}\right) \mid s\right\}$ and $0 \leq \mathbb{E}\left\{\theta r_{M} D\left(r_{M}\right)-c \mid s\right\} ; L$ invests when $\mathbb{E}\{\theta W(0)-k \mid s\} \geq \mathbb{E}\left\{\theta W\left(r_{M}\right) \mid s\right\}$, or $\mathbb{E}\{\theta W(0)-k \mid s\} \geq 0$ and $0>\mathbb{E}\left\{\theta r_{M} D\left(r_{M}\right)-c \mid s\right\}$.

Regime $D$ generates more investment than regimes $I$ and $D$. Welfare is larger than in regime $L$ because $I$ invests only when it brings more welfare than $L$. It is larger than in regime $I$ because $L$ invests only when it brings more welfare.

In the current more general setting, Regime $D$ may no longer implement the first-best allocation as the access charge chosen by the regulator may not be the best access charge given the information set of the incumbent. The regulator may then try to improve the outcome by relying on an incentive regulation scheme. We shall not pursue this line as our main goal is, rather, to evaluate when allowing duplication is inefficient. ${ }^{6}$

\section{Curbing local activism to reduce inefficient crowding- out}

We now consider that the regulator lacks information about the local authority's cost of building its own network. However, $k$ is known both by the incumbent and the local authority of that district. This highlights the fact that, at the time of devising the national regulatory scheme, the regulator may lack knowledge about the local authorities' opportunity cost for the services.

Moreover, the following assumption is now made throughout this section: The regulated access charge $r$ does not depend on the level of demand $\theta$ that prevails in the district. In our framework, this is a short-cut to model the fact that in network industries the pricing of infrastructure access by the incumbent is often required to be uniform across the national territory (the so-called perequation scheme). Alternatively, $R$ may lack reliable knowledge about the level of demand that prevails in the district at the time of deciding the national access tariff.

\footnotetext{
${ }^{6}$ See our companion paper for an analysis of the issues raised by the possibilities of contracting between the incumbent and the local authority.
} 
To streamline the analysis, consider that over the relevant range of demand parameter the regulated access tariff is such that the incumbent makes profit if it invests and is not duplicated: $\operatorname{\theta r} D(r) \geq c$. This therefore implies that there is a conflict of interest between the national regulator and the local authorities, i.e. their objectives are no longer perfectly congruent.

The conjunction of these two assumptions makes the determination of the optimal regulatory regime a non-trivial issue.

\subsection{Regimes of authorization}

If the local authority's cost were observable ex ante ${ }^{7}$ by the regulator, then $R$ has simply to establish an ex ante list of the district were duplication is allowed or not. A district where $k$ is such that $\theta W(0)-k \geq \theta W(r)+\theta r D(r)-c$ is allowed to duplicate the incumbent's investment; otherwise, the local authority is not allowed to duplicate. The incumbent then refrains from investing in those cases where it anticipates that the local authority will duplicate. Symmetric information between $R$ and $I$ implies that no inefficient duplication arises at equilibrium. For a given access tariff $r$, this ex ante rule implements the optimal outcome.

Proposition 3 When the regulator knows ex ante the local authority's investment cost, for a given access price $r$ the optimal allocation can be implemented by establishing a regime of 'ex ante authorization', i.e. an ex ante list of the districts which are allowed or not to duplicate the incumbent's network.

In many cases, though, the regulator may lack such a knowledge about the local authority's investment cost ex ante but that opportunity cost can be assessed ex post, i.e. $k$ is not observable ex ante, but is verifiable ex post. Three types of direct intervention can be envisioned:

- The regulator can systematically allow duplication, i.e. Regime $D$. Given that we focus on cases where $\operatorname{\theta r} D(r) \geq c$, the local authority duplicates when $\theta W(0)-k \geq \theta W(r)$.

- The regulator can implement a regime of 'ex post authorization' (EPA), i.e. decide whether to authorize duplication only once the local authority's investment cost can be ascertained. In that case, duplication is allowed ex post provided that $\theta W(0)-k-c \geq$ $\theta W(r)+\theta r D(r)-c$ or $\theta W(0)-k \geq \theta W(r)+\theta r D(r)$.

- The regulator can systematically ban duplication, i.e. Regime $M$. Clearly, this regime is dominated by Regime EPA.

Neither Regime $D$ nor Regime EPA do implement the socially desirable outcome. However, none of these regimes dominate the other one. Regime $D$ leads to excessive duplication

\footnotetext{
${ }^{7}$ That is, before the investment decision by the incumbent.
} 
by the local authority, which in turn yields an inefficient crowding-out of the incumbent's investment. Indeed, the local authority cares only about the welfare of its constituency and does not account for the incumbent's foregone profit $\operatorname{\theta r} D(r)-c$. Regime $E P A$ yields not enough duplication by the local authority because the incumbent tends to invest too frequently, which reduces the ex post gain associated to the intervention by the local authority.

$>$ From an ex ante perspective, the welfare difference between Regime $D$ and Regime $E P A$ writes as follows (with $\hat{k}(r)-\theta r D(r)=\theta W(0)-[\theta W(r)+\theta r D(r)] \geq 0$ ):

$$
\left.W_{D}-W_{E P A}=\int_{\hat{k}(r)-\theta r D(r)}^{\hat{k}(r)}\{\hat{k}(r)-k-[\theta r D(r)-c)]\right\} d F(k) .
$$

When $r$ is equal to $r_{b b}$, as is the case in our benchmark, Regime $D$ dominates. Otherwise, the comparison is ambiguous and one regime or the other can be optimal.

\subsection{Constraining the local authority's intervention}

The previous subsection has highlighted the difficulty to cope with the issue of duplication through regimes of authorization. Moreover, by its mere design, Regime EPA requires to be capable to assess at some point the local authority's cost, an assumption which may not be satisfied in practice. Indeed, this cost reflects local taxation and public spending considerations, as well as public debt management and political considerations. Based on this observation, we now consider two other regulatory instruments that supplement a regime with full authorization of duplication.

Consider the requirement that the incumbent be reimbursed a fraction $\alpha \in\{0,1\}$ of its investment cost when duplicated by the local authority. ${ }^{8}$ Moreover, if duplication arises, then the regulator can impose a price-floor on the local authority's network: that is, the price to access $L$ 's infrastructure cannot be lower than $l \leq r .{ }^{9}$ Thus, the incumbent decides to invest as long as it expects not to be duplicated and the local authority duplicates when $k \leq \theta[W(l)+l D(l)-W(r)]-\alpha c$. Expected welfare is thus given by:

$$
\begin{aligned}
\tilde{W}(r, \alpha, l)= & \int_{\underline{k}}^{\theta[W(l)+l D(l)-W(r)]-\alpha c}[\theta W(l)+\theta l D(l)-k] d F(k) \\
& +\int_{\theta[W(l)+l D(l)-W(r)]-\alpha c}^{+\infty}[\theta W(r)+\theta r D(r)-c] d F(k) .
\end{aligned}
$$

The determination of the optimal price-floor and reimbursement policies $\left(\alpha^{*}, l^{*}\right)$ is not enlightening. Let us rather determine some properties of the optimal policies.

Starting from Regime $D$ with no reimbursement policy, implementing a price-floor is

\footnotetext{
${ }^{8} \mathrm{We}$ assume here that the incumbent doesn't invest when it anticipates to be inactive. Otherwise, $\alpha$ should be slightly below 1 .

${ }^{9}$ As it should be clear, a price-cap has no bite in our context.
} 
optimal if:

$$
\frac{\partial \tilde{W}}{\partial l}(r, \alpha=0, l=0) \propto \theta r D(r)-c-\frac{F(\hat{k}(r))}{f(\hat{k}(r))}>0 .
$$

Imposing a price-floor has both a cost and a benefit. The cost is that the service is provided less efficiently by the local authority when it invests and it is incurred with probability $F(\hat{k})$. The benefit is that there is less inefficient public investment, where the level of inefficiency is $I^{\prime} s$ profit and the marginal effect is captured by the density. A positive price floor is then optimal only if the inefficiency in investment decisions is large. ${ }^{10}$ Notice that, given that $\hat{k}(r)=\theta[W(0)-W(r)] \geq \theta r D(r)-c$, a necessary condition for (5) to hold is that $(\hat{k}-c) f(\hat{k})>F(\hat{k})$.

Whether a price-floor policy is optimal depends on the distribution of the local authority's cost of intervention. In particular it is not optimal when the incumbent profit from investing is small.

In the subsequent analysis, we neglect the possibility of implementing a price-floor (and expressions are modified accordingly) since this not be the most relevant policy in the case of telecoms.

Starting from Regime $D$, implementing a full reimbursement policy is optimal if:

$$
\begin{aligned}
\tilde{W}(r, \alpha=1, l=0)-\tilde{W}(r, \alpha=0, l=0) \\
=-\int_{\theta[W(0)-W(r)]-c}^{\theta[W(0)-W(r)]}\{\theta W(0)-k-[\theta W(r)+\theta r D(r)-c]\} d F(k) .
\end{aligned}
$$

Condition (6) shows that when the regulated access price leaves the incumbent with no profit, then a reimbursement policy is not warranted. A regime with authorization of duplication is optimal in that case.

Complications arise when the regulated access tariff departs from this break-even point, which is the case if the regulator satisfies a uniform pricing constraint. For a district with a large demand, so that the incumbent profit tends to be large in that district, a reimbursement rule tends to be optimal.

Since revenue $\operatorname{\theta rD}(r)$ is larger than cost $c$ for investment to occur, a reimbursement policy leads to more duplication than an ex post authorization regime. Interestingly, the welfare difference between a duplication regime and an ex post authorization regime rewrites as follows:

$$
\tilde{W}(r, \alpha=1)-W_{E P A}=\int_{\theta[W(0)-W(r)]-\theta r D(r)}^{\theta[W(0)-W(r)]-c}\{\theta W(0)-k-[\theta W(r)+\theta r D(r)-c]\} d F(k) .
$$

The right-hand side of (7) is always positive. Therefore, we immediately obtain:

\footnotetext{
${ }^{10}$ The condition(5) is sufficient if the hazard rate $F(.) / f($.$) is increasing.$
} 
Proposition 4 A regime of ex post authorization is dominated by a regime with duplication and a reimbursement policy. However, if a regime with duplication is chosen, then a reimbursement policy is not always optimal.

Proof. The integrand term in the RHS of equation (7) is non-zero only if $c<\theta[W(0)-W(r)]-$ $c$ since $F(c)=0$. Suppose this holds. Then the value in the integrand is larger than $2 c-\operatorname{\theta r} D(r)>0$. Thus the integrand is positive.

Notice that this conclusion for the full reimbursement policy relies on the assumption that public transfers involve no social cost. If public funds imply distortions and social inefficiencies, a reimbursement policy will imply a cost $\alpha c$ for the collectivity where $\alpha>1$ and a deadweight loss which would tend to favour the regime of ex post authorization whenever it is feasible.

\section{Duplication risk and risk premium}

This section is devoted to the analysis of our game taking into account, again, some asymmetries of information between the incumbent and the local authority. However, the informational gap is now between, on one side, the regulator and the incumbent, and, on the other side, the local authority. To keep consistency with the previous section, assume that only $L$ does know its investment cost $k$. Note that superior information of the incumbent, for instance on the level of demand, would not invalidate our results as $I$ would still be able to anticipate the behavior of $L$. Therefore, we keep assuming that the level of demand $\theta$ is public information.

Without anticipating too much on the analysis, the crucial difference between this informational setting and the one developed in the previous section is that, now, the incumbent cannot perfectly foresee the local authority's duplication decision. Duplication may then arise with a strictly positive probability at equilibrium, leading the regulator to compensate the incumbent for this risk in order to stimulate private investment.

When $k \leq \hat{k}(r)$, the local authority bypasses the existing network and builds its own public network. Assuming duplication is authorized, the regulator has two options: trigger investment by the incumbent firm, or not.

In the first case, define the access price $r_{D}$ such that the incumbent is just indifferent between building a network or not, that is:

$$
\left[1-F\left(\hat{k}\left(r_{D}\right)\right)\right] \theta r_{D} D\left(r_{D}\right)=c .
$$

The regulator then sets $r=r_{D}$ to induce investment. Of course $r_{D}$ may not exist, in which case Regime $D$ is not feasible and resume to regime $L$. To focus on the interesting cases, we assume from now on that $r_{D}$ exists. 
Expected welfare in the regime with duplication writes as follows:

$$
\begin{aligned}
W_{D} & =\int_{\underline{k}}^{\hat{k}\left(r_{D}\right)}[\theta W(0)-k-c] d F(k)+\int_{\hat{k}\left(r_{D}\right)}^{+\infty}\left[\theta W\left(r_{D}\right)+\theta r_{D} D\left(r_{D}\right)-c\right] d F(k), \\
& =\int_{\underline{k}}^{\hat{k}\left(r_{D}\right)}[\theta W(0)-k] d F(k)+\int_{\hat{k}\left(r_{D}\right)}^{+\infty} \theta W\left(r_{D}\right) d F(k) .
\end{aligned}
$$

Another option is to discourage the incumbent from investing by setting too low an access price (for instance $r<r_{D}$ ); only the local authority does invest, provided its cost parameter is sufficiently low, or, formally, provided that $k \leq \theta W(0)$. Expected welfare in that case can thus be written as follows: $W_{L}=\int_{\underline{k}}^{\theta W(0)}[\theta W(0)-k] d F(k)$.

Interestingly, the comparison of the two regulatory policies turns out to be unambiguous:

Proposition 5 Under asymmetric information on the local authority's cost parameter only, the regulator always prefers to induce investment by the incumbent with duplication than to prevent investment by the incumbent, i.e. Regime $D$ dominates Regime $L$ (when it induces investment by the incumbent).

Proof. Suppose $r_{D}$ exists and rewrite $W_{D}$ as follows:

$$
\begin{aligned}
W_{D} & =\int_{\underline{k}}^{\hat{k}\left(r_{D}\right)}[\theta W(0)-k] d F(k)+\int_{\hat{k}\left(r_{D}\right)}^{+\infty} \theta W\left(r_{D}\right) d F(k), \\
& =W_{L}+\int_{\hat{k}\left(r_{D}\right)}^{\theta W(0)}\left[\theta W\left(r_{D}\right)-\theta W(0)+k\right] d F(k)+\int_{\theta W(0)}^{+\infty} \theta W\left(r_{D}\right) d F(k) .
\end{aligned}
$$

Obviously, the second integral in the last expression is positive. Notice also that, for any $r$, $\hat{k}(r) \leq \theta W(0)$ and that $\hat{k}(r) \leq k \leq \theta W(0)$ is equivalent to $\theta[W(0)-W(r)] \leq k \leq \theta W(0)$. Therefore, the first integral is also positive. We thus conclude that $W_{D}>W_{L}$.

The next step is to determine when it is optimal to ban the intervention of the local authority (Regime $M$ ). Obviously, there is a trade-off. On the one hand, the intervention of the local authority arises provided that its cost parameter is not too high and allows to benefit from a reduced final price. On the other hand, since the incumbent can no longer perfectly anticipate the local authority's ex post intervention due to asymmetric information, the incumbent's ex ante incentive to invest in the network upgrade degrades, thereby forcing the regulator to increase the access price.

Formally, if there is a ban on the intervention by the local authority, the regulator provides the incumbent with the incentives to upgrade the network by setting the access price $r_{M}=r_{b b}$ (such that: $\theta r_{b b} D\left(r_{b b}\right)-c=0$ ). Expected welfare is thus given by: $W_{M}=\theta W\left(r_{b b}\right)$.

Comparing $W_{M}$ and $W_{D}$, we obtain the following conclusion:

Proposition 6 When there is asymmetric information on the local authority's cost parameter only, it is preferable to ban duplication and induce investment by the incumbent (strictly 
if duplication arises with positive probability in Regime $D)$, i.e. regime $M$ dominates regime $D$.

Proof. See Appendix A.1.

Proposition 6 states that when the incumbent invests and there is some duplication, it would be better to simply induce investment with no duplication. The result is due to the fact that inducing the incumbent to invest requires compensating it for the risk of duplication by raising the access charge. Compared to setting $r_{b b}$ with no duplication, this increase in access charge offsets any benefits that may arise from the local authority's intervention.

As opposed to the previous case of symmetric information, duplication is not always efficient because $r_{D}>r_{b b}$. There exists a range of cost $k$ where duplication occurs while it would be more efficient to let the incumbent invest but with the break-even access charge $r_{b b}$.

The general conclusion is thus that with symmetric information between the regulator and the incumbent, but imperfect information on the local authority's opportunity cost of intervention, the optimal regulation takes the following form:

- For low values of cost $c$, the incumbent invests with an access charge set so as to just generate fair return on investment and local intervention is forbidden.

- For large $c$, the local authority invests.

The threshold $\hat{c}$ for which the local authority is indifferent between investing or not is solution of:

$$
\theta W\left(r_{b b}\right)=\mathbb{E}\{\max (\theta W(0)-k, 0)\} .
$$

It is larger than the maximal value for which regime $D$ would be feasible:

$$
\hat{c}>\max _{r}[1-F(\hat{k}(r))] \operatorname{\theta r} D(r) .
$$

\section{Technological externalities and electronic dumping}

In this section, we introduce another relevant ingredient which may lead to inefficient duplication: externalities between regions. We focus on two kinds of externalities, technological ones and externalities linked to a form of (fiscal) competition between districts.

To discuss this we consider the case of two districts, 1 and 2; each district $i$ is characterized by parameters $\theta_{i}$ and $c_{i}$ where $c_{i}$ is interpreted as the cost for the incumbent to develop the infrastructure in district $i$ alone. We assume that the demand-adjusted cost is larger in district 1 than in district 2: $\frac{c_{2}}{\theta_{2}} \leq \frac{c_{1}}{\theta_{1}}$.

To simplify matters we focus on the externality exerted by duplication in district 2 on district 1 by assuming that there is no local authority in district 1 that can invest in infrastructure. Moreover we assume that the regulator maximizes total consumers surplus; 
this allows focusing on inefficiencies not related to conflicting objectives between the regulator and the local authorities as a whole. The local authority in district 2 maximizes the surplus of the initial population in its own district: it considers only the immobile agents even if, ex post, mobile agents turn out to settle in district 2. This assumption builds on the fact the local authority is a representative (possibly elected) of the inhabitants of the district, and, therefore, is subject to the influence of the agents living in the district at the moment of making choices.

We work under the assumption of a unique access charge nationwide. Indeed, the common practice, in particular for broadband access, is to have a unique access charge across all districts, referred to as a universal service obligation or USO. Note that allowing the local authority to intervene on market conditions amounts to deviating from a general principle of USO, for this implies differential access conditions. Although our objective in this paper is not to develop the analysis of USO we want to stress that it is not clear whether there is any rational for USO once local intervention is allowed. ${ }^{11}$

The timing is as follows: the regulator chooses an access price $r$; the incumbent decides whether to undertake the network upgrade in each district; the local authority in district 2 decides to invest of not in the infrastructure.

Duplication may prevent the incumbent from exploiting scale economy at the level of the national territory, thereby creating inefficiencies. Indeed, it was assumed so far that the cost of providing the infrastructure was fixed in each district. In practice, though, there are common fixed costs in developing infrastructures. To illustrate this effect, we consider the case of two districts with a technological externality. Formally, we assume that $c_{i}$ is the cost of developing the infrastructure in district $i$ only, while $c_{1}+c_{2}-\delta$ is the cost of developing the infrastructure in both districts. Thus, $\delta$ can be seen as a common fixed cost.

Consider first regime $M$. Then, the incumbent builds the infrastructure in both districts if:

$$
\begin{aligned}
\left(\theta_{1}+\theta_{2}\right) r D(r)-\left(c_{1}+c_{2}-\delta\right) & \geq 0 \\
\theta_{1} r D(r)-\left(c_{1}-\delta\right) & \geq 0 \\
\theta_{2} r D(r)-\left(c_{2}-\delta\right) & \geq 0
\end{aligned}
$$

The smallest regulated access charge $r_{M}$ is then solution of:

$$
r_{M} D\left(r_{M}\right)=\max \left\{\frac{c_{1}-\delta}{\theta_{1}}, \frac{c_{1}+c_{2}-\delta}{\theta_{1}+\theta_{2}}\right\}<\frac{c_{1}}{\theta_{1}} .
$$

Consider now regime $D$. Then, duplication occurs at $r=r_{M}$ if $k_{2}<\theta_{2}\left[W(0)-W\left(r_{M}\right)\right]$. When this is the case, the regulator needs to set a price $r=r_{D}>r_{M}$ such that $\theta_{1} r_{D} D\left(r_{D}\right)=$

\footnotetext{
${ }^{11}$ The problem is similar with the one of USO under liberalization and competition, namely one of creamskimming.
} 
$c_{1}$. Thus, duplication forces to raise the price in district 1 to compensate for the foregone scale economies. Then we obtain:

Proposition 7 With scale economy, there is a range of the local authority's cost parameter for which it is optimal to ban duplication.

Proof. Duplication is efficient if $\theta_{1} W\left(r_{D}\right)+\theta_{2} W(0)-k_{2}>\left(\theta_{1}+\theta_{2}\right) W\left(r_{M}\right)$. Thus, duplication should be forbidden when: $\theta_{2}\left[W(0)-W\left(r_{M}\right)\right]+\theta_{1}\left[W\left(r_{D}\right)-W\left(r_{M}\right)\right]<k_{2}<$ $\theta_{2}\left[W(0)-W\left(r_{M}\right)\right]$.

Another potential source of inefficiency is grounded in the competition between districts. To get an idea on the possible interactions between districts in their investment and pricing choices, let us amend our framework as follows.

Suppose that on top of the population living in each district, there is a mass of mobile agents, either wealthy consumers or firms, who choose their location according to the proposals made by the districts. To keep things simple, we assume that those mobile agents have the same demand as the standard ones but with a coefficient $\hat{\theta}$ and that those agents are initially located in district $1 .{ }^{12}$ Moreover, we take the assumption that the mobile agents cannot modify the rankings of the cost/demand ratio across districts, i.e. $\frac{c_{2}}{\theta_{2}} \leq \frac{c_{1}}{\theta_{1}+\hat{\theta}} \leq \frac{c_{1}}{\theta_{1}}$.

Since the policy chosen in each district may trigger some mobility, there are some externalities between districts. More precisely, if there is no migration from the first to the second district, the average cost for a given access price $r$ is given by $\frac{c_{1}}{\left(\theta_{1}+\hat{\theta}\right) D(r)}$. In case of mobility, this costs rises to $\frac{c_{1}}{\theta_{1} D(r)}$. Therefore, the choice of the regulated access charge must take into account the ex post choice of the district 2 and of the mobile agents. This may be another rationale for banning or allowing duplication.

To focus on the most relevant cases, assume from now on that the regulator wants to ensure that investment takes place in district 1. In the absence of a public decision-maker in district 1 , this means that the incumbent must be given incentives to invest in this district.

In a regime without duplication, setting an access price $r_{M}$ such that $\left(\theta_{1}+\hat{\theta}\right) r_{M} D\left(r_{M}\right)=c_{1}$ triggers private investment both in district 1 and in district 2. Consumers' welfare is then given by:

$$
S\left(r_{M}\right)=\left(\theta_{1}+\hat{\theta}+\theta_{2}\right) W\left(r_{M}\right) .
$$

Any other regulated access price is either unnecessarily high or triggers private investment only in district 2 (together with mobility of the mobile consumers).

Assume now that duplication is allowed. Given a regulated access price $r$, the local authority in district 2 decides to duplicate the incumbent provided that:

$$
\max _{a_{2} \leq r} \theta_{2} W\left(a_{2}\right)+\left(\theta_{2}+\hat{\theta}\right) a_{2} D\left(a_{2}\right)-k_{2} \geq \theta_{2} W(r) .
$$

\footnotetext{
${ }^{12}$ Mobile agents in district 2 would not migrate and are therefore aggregated in the demand in disctrict 2.
} 
Define consequently $\hat{k}_{2}(r)$ as the threshold of the local authority's cost below which duplication occurs and $\hat{a}_{2}(r)$ as the access price implemented in district 2 in that case:

$$
\hat{a}_{2}(r)=\min \left\{r, \frac{\theta_{2}}{\theta_{2}+\hat{\theta}} \frac{D\left(\hat{a}_{2}\right)}{-D^{\prime}\left(\hat{a}_{2}\right)}\right\} .
$$

As above, if $k_{2}$ is known to be below $\hat{k}_{2}\left(r_{M}\right)$, a regime $D$ requires to set the price in district 1 at the level $r_{D}>r_{M}$ such that $\theta_{1} r_{D} D\left(r_{D}\right)=c_{1}$. Allowing duplication involves, on the one hand, a higher regulated access price on district 1 because the firm faces a risk of duplication, and, on the other hand, a lower access price in district 2, which benefits the mobile citizens on top of those living initially in district 2 . While the fact that mobile citizens benefit makes a difference, we still obtain:

Proposition 8 With a mobile population, there is a range of the local authority's cost parameter for which it is optimal to ban duplication.

Proof. Duplication is efficient if

$\theta_{1} W\left(r_{D}\right)+\left(\theta_{2}+\hat{\theta}\right) W\left(\hat{a}_{2}\left(r_{D}\right)\right)+\left(\theta_{2}+\hat{\theta}\right) \hat{a}_{2}\left(r_{D}\right) D\left(\hat{a}_{2}\left(r_{D}\right)\right)-k_{2}>\left(\theta_{1}+\theta_{2}+\hat{\theta}\right) W\left(r_{M}\right)$.

Thus, duplication should be forbidden when:

$$
\begin{aligned}
& \theta_{1} W\left(r_{D}\right)+\left(\theta_{2}+\hat{\theta}\right) W\left(\hat{a}_{2}\left(r_{D}\right)\right)+\left(\theta_{2}+\hat{\theta}\right) \hat{a}_{2}\left(r_{D}\right) D\left(\hat{a}_{2}\left(r_{D}\right)\right)-\left(\theta_{1}+\theta_{2}+\hat{\theta}\right) W\left(r_{M}\right) \\
< & k_{2}<\theta_{2} W\left(\hat{a}_{2}\left(r_{M}\right)\right)+\left(\theta_{2}+\hat{\theta}\right) \hat{a}_{2}\left(r_{M}\right) D\left(\hat{a}_{2}\left(r_{M}\right)\right)-\theta_{2} W\left(r_{M}\right)
\end{aligned}
$$

Then we have

$$
\begin{aligned}
& \theta_{1} W\left(r_{D}\right)+\left(\theta_{2}+\hat{\theta}\right) W\left(\hat{a}_{2}\left(r_{D}\right)\right)+\left(\theta_{2}+\hat{\theta}\right) \hat{a}_{2}\left(r_{D}\right) D\left(\hat{a}_{2}\left(r_{D}\right)\right)-\left(\theta_{1}+\theta_{2}+\hat{\theta}\right) W\left(r_{M}\right) \\
< & \theta_{2} W\left(\hat{a}_{2}\left(r_{M}\right)\right)+\left(\theta_{2}+\hat{\theta}\right) \hat{a}_{2}\left(r_{M}\right) D\left(\hat{a}_{2}\left(r_{M}\right)\right)-\theta_{2} W\left(r_{M}\right) \\
& +\theta_{1} W\left(r_{D}\right)+\hat{\theta} W(0)-\left(\theta_{1}+\hat{\theta}\right) W\left(r_{M}\right)
\end{aligned}
$$

Remind that $\theta_{1} r_{D} D\left(r_{D}\right)+\hat{\theta} \cdot 0=\left(\theta_{1}+\hat{\theta}\right) r_{M} D\left(r_{M}\right)=c_{1}$. But our assumption on $r$ implies that the welfare $W$ is a concave of the revenue $r D(r)$ on the relevant range of price. Thus $\theta_{1} W\left(r_{D}\right)+\hat{\theta} W(0)-\left(\theta_{1}+\hat{\theta}\right) W\left(r_{M}\right)<0$ which implies that the condition is satisfied for an interval of $k_{2}$.

More generally, when the regulator is uncertain about the duplication decision by the local authority, the only way to trigger private investment in district 1 is to set the regulated access price at level $r_{D}$ : even if mobile citizens move to district 2 , the incumbent is then willing to undertake the investment in district 1 . Clearly when the regulator is eager to preserve investment in district 1, a ban on duplication is optimal when the gain from duplication is 
small, thus when the likelihood of duplication is small or local investment cost lies in the interval identified above.

\section{Conclusion}

Investment by local government may help fostering fast development of new infrastructures and reaching a large coverage of population. As such it may be an important element of public policies aiming at reducing the potential gap between highly competitive zones and less competitive ones. Still public policy should guard itself from potential crowding out of efficient private investment, which may occur when public investment is not restricted to areas where private investment is deficient.

Our paper helps understanding the issues at stake by focusing on situations where conditions for competition are not met, but it is not obvious whether a regulated private monopoly dominates a local public investment. Thus our conclusions are valid in such grey areas where competition is not effective enough.

We identified three key dimensions that should be considered with special assumptions when designing rules governing the intervention of local authorities. One is the risk born by private investors, that may refrain them from investing. Costly ex-ante weakening of regulation is then required to restore investment incentives. Our results suggest that when this is the sole distortion, the benefits of local intervention doesn't outweigh the cost of lenient regulation.

Other issues relate to differences between the motives of the local authorities and the social welfare. Such differences in objectives may result from a lack of appraisal of foregone profits at the local level as in section 3, or from various forms of externalities as in section 6 . In particular, competition between collectivities trying to propose the best environment for mobile factors may lead to excessive investment. In discussing these issues we ignored political economy or prestige considerations that may lead politicians to invest excessively in advanced technologies at the expense of less rewarding by more useful local goods. Our discussion of the regulation with conflicting objectives doesn't clearly support banning duplication but rather suggests that some form of control and limits on the local interventions may help improve efficiency. 


\section{A Appendix}

\section{A.1 Proof of Proposition 6}

Of course, if there is no investment in Regime $D$, banning duplication has no effect since the regulatory choice is only between allowing the incumbent to invest with no duplication, or no investment by the incumbent and investment by the local authority. Assume from now on that there is investment in Regime $D$.

One has to show that $W_{M}-W_{D}$ is always positive when duplication arises with a strictly positive probability under Regime $D$. We have:

$$
K(c)=W_{M}-W_{D}=\theta W\left(r_{b b}\right)-\left[\int_{\underline{k}}^{\hat{k}\left(r_{D}\right)}[\theta W(0)-k] d F(k)+\int_{\hat{k}\left(r_{D}\right)}^{+\infty} \theta W\left(r_{D}\right) d F(k)\right] .
$$

Note first that when $c=0$, then $r_{D}=r_{b b}=0$ and $\hat{k}\left(r_{D}\right)=0$. Therefore, $K(0)=0$.

Now let us differentiate $K($.$) with respect to c$ :

$$
K^{\prime}(c)=-\theta D\left(r_{b b}\right) \frac{\partial r_{b b}}{\partial c}+\theta D\left(r_{D}\right) \frac{\partial r_{D}}{\partial c}\left[1-F\left(\hat{k}\left(r_{D}\right)\right)\right] .
$$

Remark that $r_{b b}$ and $r_{D}$ are increasing functions of $c$. Let us consider first the definition of $r_{b b}$. It is the smallest solution of the following relationship: $\theta r_{b b} D\left(r_{b b}\right)-c=0$. It exists for $c$ less than the monopoly profit. Using the implicit function theorem, it is therefore direct to conclude that:

$$
\frac{\partial r_{b b}}{\partial c}=\frac{1}{\theta\left[D\left(r_{b b}\right)+r_{b b} D^{\prime}\left(r_{b b}\right)\right]}>0
$$

since $r_{b b} \leq a_{m}=\arg \max _{a} a D(a)$.

Similarly, $r_{D}$ is defined as the smallest solution of: $\left[1-F\left(\hat{k}\left(r_{D}\right)\right] \theta r_{D} D\left(r_{D}\right)-c=0\right.$. Since for $c=0$ the solution is $r_{D}=0$, and using a continuity argument, there are solutions to the previous equation for small $c$ and a straightforward argument shows that the smallest one is increasing with $c$. Notice that $r_{b b}$ exists if $r_{D}$ exists.

There may be upward discontinuities of $r_{D}$. At such point $K($.$) has an upward disconti-$ nuity. Now, where $r_{D}$ is continuous, it is differentiable and the derivative of the gross profit function is increasing (since $a_{D} \leq a_{m}$ ). More precisely, we have:

$$
\frac{\partial r_{D}}{\partial c}=\frac{1}{\theta\left[D\left(r_{D}\right)\left(1-F\left(\hat{k}\left(r_{D}\right)\right)\right)+r_{D} \frac{\partial}{\partial r}\left(\left.D(r)(1-F(\hat{k}(r)))\right|_{r=r_{D}}\right]\right.}>0 .
$$

We can now rewrite the expression for $K^{\prime}(c)$ as follows:

$$
K^{\prime}(c)=\frac{-D\left(r_{b b}\right)}{D\left(r_{b b}\right)+r_{b b} D^{\prime}\left(r_{b b}\right)}+\frac{D\left(r_{D}\right)(1-F(\hat{k}))}{D\left(r_{D}\right)\left(1-F\left(\hat{k}\left(r_{D}\right)\right)\right)+\left.r_{D} \frac{\partial}{\partial r}(D(r)(1-F(\hat{k}(r))))\right|_{r=r_{D}}} .
$$


Now, using the positivity of the denominator, we can state that:

$$
\begin{aligned}
K^{\prime}(c) \geq 0 & \Leftrightarrow \frac{D\left(r_{b b}\right)+r_{b b} D^{\prime}\left(r_{b b}\right)}{D\left(r_{b b}\right)} \geq \frac{D\left(r_{D}\right)\left(1-F\left(\hat{k}\left(r_{D}\right)\right)\right)+\left.r_{D} \frac{\partial}{\partial r}(D(r)(1-F(\hat{k}(r))))\right|_{r=r_{D}}}{D\left(r_{D}\right)\left(1-F\left(\hat{k}\left(r_{D}\right)\right)\right)}, \\
& \Leftrightarrow 1-\varepsilon\left(r_{b b}\right) \geq 1+\left.\frac{\partial}{\partial r}(D(r)(1-F(\hat{k}(r))))\right|_{r=r_{D}} \frac{r_{D}}{D\left(r_{D}\right)\left(1-F\left(\hat{k}\left(r_{D}\right)\right)\right)}, \\
& \Leftrightarrow-\varepsilon\left(r_{b b}\right) \geq \frac{r_{D}\left[D^{\prime}\left(r_{D}\right)\left(1-F\left(\hat{k}\left(r_{D}\right)\right)\right)+\left.D\left(r_{D}\right) \frac{\partial}{\partial r}\left(1-F\left(\hat{k}\left(r_{D}\right)\right)\right)\right|_{r=r_{D}}\right]}{D\left(r_{D}\right)\left(1-F\left(\hat{k}\left(r_{D}\right)\right)\right)}, \\
& \Leftrightarrow-\varepsilon\left(r_{b b}\right) \geq-\varepsilon\left(r_{D}\right)+r_{D} \frac{\left.\frac{\partial}{\partial r}(1-F(\hat{k}(r)))\right|_{r=r_{D}} .}{\left(1-F\left(\hat{k}\left(r_{D}\right)\right)\right)}
\end{aligned}
$$

Since $r_{b b} \leq r_{D}$ and $\varepsilon($.$) is increasing, -\varepsilon\left(r_{b b}\right) \geq-\varepsilon\left(r_{D}\right)$. Moreover:

$$
\left.\frac{\partial}{\partial r}(1-F(\hat{k}(r)))\right|_{r=r_{D}}=-\frac{d \hat{k}}{\partial r}\left(r_{D}\right) f\left(\hat{k}\left(r_{D}\right)\right)=-\theta D\left(r_{D}\right) f\left(\hat{k}\left(r_{D}\right)\right) \leq 0 .
$$

So $K^{\prime}(c) \geq 0$ when $r_{D}$ is continuous. Thus $K(c)$ is nondecreasing with $c$. Using the fact that $K(0)=0$, we obtain that $K(c)$ is non-negative on its domain. 


\section{References}

[1] ARCEP, 2008, "L’intervention des Collectivités Territoriales dans les Télécommunications."

[2] Caillaud B., and J. Tirole (2004). "Essential facility financing and market structure", Journal of Public Economics, Vol. 88, 667-694.

[3] Dana J.D., and K. Spier (1994). "Designing a private industry: Government auctions with endogenous market structure", Journal of Public Economics, Vol. 53, 667-694.

[4] EC, 2009, "Community Guidelines for the Application of State Aid Rules in Relation to Rapid Deployment of Broadband Networks," Public consultation on the Commission's Broadband Guidelines on the application of EU state aid rules to public funding of broadband networks.

[5] Jullien B., Pouyet J. and W. Sand-Zantman 2009. "Contracting and Regulation under the threat of duplication", IDEI.

[6] Laffont, J.-J. and J. Tirole, 2001, Competition in telecommunications, MIT Press.

[7] Ofcom, 2007, "Future Broadband. Policy approach to next generation access." 